\title{
Modelo para Dispersão de Poluentes na Atmosfera com Condições de Contorno Parcialmente Reflexivas
}

\author{
Jaqueline Fischer Loeck Bardo Bodmann Marco Vilhena \\ Programa de Pós-Graduação em Engenharia Mecânica, UFRGS, \\ 90050-170, Porto Alegre, RS \\ E-mail: emaildajaque@gmail.com, bardo.bodmann@ufrgs.br, vilhena@mat.ufrgs.br
}

\section{RESUMO}

A equação de advecção-difusão modela o fenômeno de dispersão de poluentes na camada limite atmosférica. Tal equação com fechamento Fickiano para turbulência e coeficientes de difusão $K_{x}, K_{y}$ e $K_{z}$ constantes, pode ser escrita como

$$
\frac{\partial \bar{c}}{\partial t}+\bar{u} \frac{\partial \bar{c}}{\partial x}+\bar{v} \frac{\partial \bar{c}}{\partial y}+\bar{w} \frac{\partial \bar{c}}{\partial z}=K_{x} \frac{\partial^{2} \bar{c}}{\partial x^{2}}+K_{y} \frac{\partial^{2} \bar{c}}{\partial y^{2}}+K_{z} \frac{\partial^{2} \bar{c}}{\partial z^{2}}+\bar{S}
$$

onde $\bar{c}$ é a concentração média do poluente, $\bar{u}, \bar{v}$ e $\bar{w}$ são as componentes de vento médio orientados nas direções $x, y$ e $z$, respectivamente, e $\bar{S}$ é o termo fonte.

No presente trabalho, o termo fonte é descrito como uma condição inicial instantânea e, posteriormente, a solução para emissão instantânea é integrada no tempo para obter a solução para emissão contínua, que é dada por

$\bar{C}(x, y, z, t)=\frac{1}{T} \frac{Q}{\sqrt{64 \pi^{3} K_{x} K_{y} K_{z}}} \int_{0}^{t} \frac{1}{\sqrt{(t-\tau)^{3}}} e^{-\frac{\left[x-x_{0}-\bar{u}(t-\tau)\right]^{2}}{4 K_{x}(t-\tau)}} e^{-\frac{\left[y-y_{0}-\bar{v}(t-\tau)\right]^{2}}{4 K_{y}(t-\tau)}} e^{-\frac{-\left[z-H_{s}-\bar{w}(t-\tau)\right]^{2}}{4 K_{z}(t-\tau)}} d \tau$.

Tal solução é obtida através da transformada de Fourier e é válida para $z \in(-\infty, \infty)$, porém o solo e a camada limite atmosférica limitam a dispersão, de forma que o intervalo infinito deve ser mapeado para o intervalo finito $z \in\left[0, z_{i}\right]$, onde $z_{i}$ é a altura do topo da camada limite atmosférica. Para isto, serão consideradas infinitas reflexões tanto no solo quanto na camada limite. Estas reflexões podem ser representadas pelas duas sequências

$$
\left.\begin{array}{l}
H_{s} \rightarrow-H_{s}-2 n z_{i} \\
H_{s} \rightarrow H_{s}+2 n z_{i}
\end{array}\right\} \forall n \in \mathbb{Z}
$$

As sequências apresentadas acima consideram que o poluente que está dispersando, quando atingir o solo ou o topo da camada limite, será refletido por completo de volta para o domínio, sem considerar uma possível permeabilidade parcial, onde parte do material pode se infiltrar no solo ou ultrapassar a camada limite. Considerando esta permeabilidade, serão introduzidos nas sequências que consideram as reflexões os termos $\omega_{c}$ e $\omega_{s}$ que são, respectivamente, os parâmetros de reflexão na camada limite atmosférica e no solo. Estes termos descrevem a quantidade de material poluente que está sendo refletida. Desta maneira, as sequências que representam as reflexões, incluindo a permeabilidade parcial, são descritas por

$$
\left.\begin{array}{l}
H_{s} \rightarrow-\omega_{s} H_{s}-2 n \omega_{c} z_{i} \\
H_{s} \rightarrow \omega_{s} H_{s}+2 n \omega_{c} z_{i}
\end{array}\right\} \forall n \in \mathbb{Z}
$$

$\mathrm{Na}$ solução apresentada anteriormente (2), onde antes apenas era considerada a altura da fonte $H_{s}$, agora serão consideradas estas duas sequências, de maneira que a solução para emissão contínua com reflexão parcial nos contornos é dada por 


$$
\begin{aligned}
\bar{C}(x, y, z, t)= & \frac{1}{T} \frac{Q}{\sqrt{64 \pi^{3} K_{x} K_{y} K_{z}}} \int_{0}^{t}\left\{\frac{1}{\sqrt{(t-\tau)^{3}}} e^{-\frac{\left[x-x_{0}-\bar{u}(t-\tau)\right]^{2}}{4 K_{x}(t-\tau)}} e^{-\frac{\left[y-y_{0}-\bar{v}(t-\tau)\right]^{2}}{4 K_{y}(t-\tau)}}\right. \\
& \left.\times \sum_{n=-\infty}^{\infty}\left[e^{-\frac{\left[z-\omega_{s} H_{s}-2 n \omega_{c} z_{i}-\bar{w}(t-\tau)\right]^{2}}{4 K_{z} t}}+e^{-\frac{\left[z+\omega_{s} H_{s}+2 n \omega_{c} z_{i}-\bar{w}(t-\tau)\right]^{2}}{4 K_{z} t}}\right]\right\} d \tau .
\end{aligned}
$$

Os resultados obtidos foram comparados com o experimento de Hanford [2] e são preliminares, com o propósito de determinar a contribuição das reflexões na concentração obtida pelo modelo adotado. Para diferentes valores adotados para $\omega_{c}$ e $\omega_{s}$, foram obtidos números de reflexões distintos, sendo que as reflexões são repetidas até não haver mais contribuição na solução. A Figura 1 apresenta uma comparação entre as concentrações para o modelo sem reflexão e com as reflexões completas e parciais, respectivamente.

É possível observar que quando são consideradas as reflexões parciais, os valores obtidos para as concentrações se aproximam consideravelmente dos valores medidos no experimento de Hanford. Desta forma, as reflexões parciais consideram contribuições que o modelo inicial desconsidera. Além disso, foi constatado que ao considerar reflexão completa nos contornos, há uma melhora na solução, porém os resultados do modelo não são aceitáveis. Assim, o modelo que inclui estas reflexões parciais pode ser considerado mais próximo do fenômeno físico analisado em comparação aos modelos que consideram o poluente confinado entre o solo e a camada limite atmosférica, e utilizam como condição de contorno concentração ou fluxo de concentração nulos.
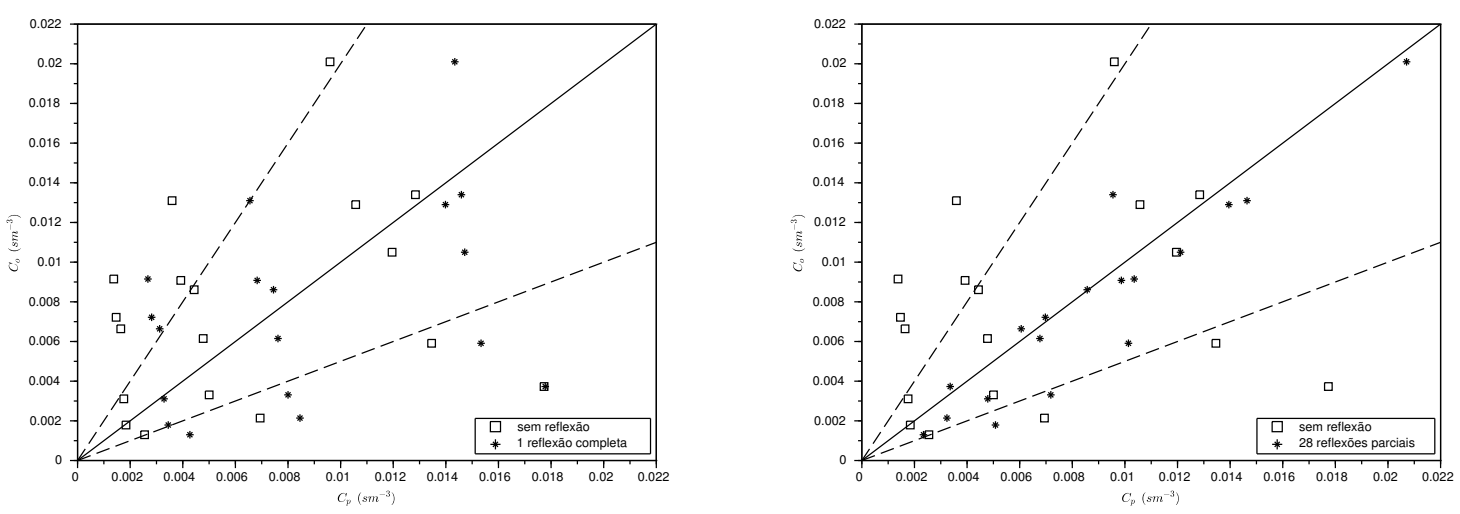

Figura 1: Gráficos de espalhamento das concentrações observadas $C_{o}$ e previstas pelo modelo $C_{p}$ sem refexão e com reflexões parciais utilizando-se os parâmetros: a) $\omega_{s}=1,0$ e $\omega_{c}=1,0$ com 1 reflexão completa e b) $\omega_{s}=0,1$ e $\omega_{c}=0,01$ com 28 reflexões parciais.

Palavras-chave: equação de advecção-difusão, reflexão nos contornos, reflexão parcial

\section{Referências}

[1] S. P. Arya, "Air pollution meteorology and dispersion", Oxford University Press, New York, 1999.

[2] J. C. Doran and T. W. Horst, An evaluation of Gaussian plume depletion models with dual-tracer field measurements, Atmospheric Environment, 19 (1985) 939-951.

[3] J. H. Seinfeld and S. N. Pandis, "Atmospheric chemistry and physics: from air pollution to climate change", John Wiley \& Sons, New Jersey, 2nd edition, 2006.

[4] R. B. Stull, "An introduction to boundary layer meteorology", Kluwer Academic Publishers, Dordrecht, Holanda, 1988. 